2017-12-15

In vitro toxic effects of reduced graphene oxide nanosheets on lung cancer cells

\title{
Tabish, TA
}

http://hdl.handle.net/10026.1/13480

\subsection{8/1361-6528/aa95a8}

Nanotechnology

IOP Publishing

All content in PEARL is protected by copyright law. Author manuscripts are made available in accordance with publisher policies. Please cite only the published version using the details provided on the item record or document. In the absence of an open licence (e.g. Creative Commons), permissions for further reuse of content should be sought from the publisher or author. 


\section{In vitro toxic effects of reduced graphene oxide nanosheets on lung cancer cells}

Tanveer A. Tabish ${ }^{1}$, Md Zahidul I. Pranjol${ }^{2}$, Hasan Hayat ${ }^{1}$, Alma A. M. Rahat ${ }^{3}$, Trefa M. Abdullah², Jacqueline L. Whatmore ${ }^{*, 2}$, and Shaowei Zhang ${ }^{1, *}$

${ }^{1}$ College of Engineering, Mathematics and Physical Sciences, University of Exeter, Exeter, EX4 4QF, United Kingdom

${ }^{2}$ Institute of Biomedical and Clinical Science, University of Exeter Medical School, St Luke's Campus, EX1 2LU, United Kingdom

${ }^{3}$ Department of Computer Science, University of Exeter, Exeter, EX4 4QF, United Kingdom

Address correspondence to $\underline{\text { J.L.Whatmore@exeter.ac.uk; }} \underline{\text { s.zhang@exeter.ac.uk }}$

\section{Abstract:}

The intriguing properties of reduced graphene oxide ( $\mathrm{rGO}$ ) have paved the way for a number of potential biomedical applications such as drug delivery, tissue engineering, gene delivery and bio-sensing. Over the last decade, there have been escalating concerns regarding the possible toxic effects, behaviour and fate of rGO in living systems and environments. This paper reports on integrative chemical-biological interactions of rGO with lung cancer cells, i.e. A549 and SKMES-1, to determine its potential toxicological impacts on them, as a function of its concentration. Cell viability, early and late apoptosis and necrosis were measured to determine oxidative stress potential, and induction of apoptosis for the first time by comparing two lung cancer cells. We also showed the general trend between cell death rates and concentrations for different cell types using a Gaussian process regression model. At low concentrations, rGO was shown to significantly produce late apoptosis and necrosis rather than early apoptotic events, suggesting that it was able to disintegrate the cellular membranes in a dose dependent manner. For the toxicity exposures undertaken, late apoptosis and necrosis occurred, which was most likely resultant from limited bioavailability of unmodified rGO in lung cancer cells.

Keywords: reduced graphene oxide nanosheets, cell viability, toxicity, apoptosis, necrosis

\section{Introduction:}


The potential applications of graphene are rapidly expanding with a global industry estimated to be worth more than 1790.7 Million USD by 2020 [1], which is reflective of its wide range of application domains including, electronics, supercapacitors, energy storage and medicine [2]. The development of real-world applications of graphene is fuelled by its unique and superior properties such as high electron mobility, high mechanical strength and high specific surface area [3]. Although some effort has been made to investigate the biosafety profile of graphene, a significant lack of viable data on biocompatibility hinders the precise forecast of the potential of graphene to solve real-world clinical problems.

Pristine graphene, graphene oxide and reduced graphene oxide (rGO) have been investigated as potentially hazardous materials when used in healthcare because they could exert acute toxic effects on a wide range of living organisms including human cells, Gram-positive and Gram-negative bacteria, viruses, and plants, eukaryotic mammalian and in vivo animal models $[4,5]$. Current knowledge on their toxicological implications indicates the demand for further systematic investigations including a detailed basic physicochemical characterisation of the graphene-based materials exploited in each case. It has been demonstrated that graphene nanostructures cause harmful cellular effects when they enter the body, as they can pass through physiological barriers, encounter immune systems and trigger normal cellular responses and significantly enhance toxic potential in living systems [6]. Single and few-layered graphene having sharp edges may infiltrate cell membranes resulting in membrane damage and leakage of cytoplasmic substances. DNA damage, cell cycle arrest and oxidative stresses inside the cell are the main cytotoxicity responses to $\mathrm{GO}$ and $\mathrm{rGO}$ when they are exposed to different cell lines, which are likely due to the generation of reactive oxygen species, and deregulation of antioxidant genes [7]. The biocompatibility of graphene varies from their counterparts owing to their size, shape, lateral dimensions, high specific surface area and surface chemistry [8]. Most of the studies to date have focused mainly on the toxicity induced by pristine graphene and GO but the biocompatibility of rGO has not been fully understood. Recently, rGO has been evaluated for biological applications, for example, as drug delivery carriers, diagnostic sensors, biomarkers and antimicrobial agents [9]. However, it has been shown to cause several adverse effects in vitro including reactive oxygen species 
formation, cell apoptosis, inflammatory cytokine, loss of membrane integrity, membrane distress induced by direct contact with sharp edges of $\mathrm{rGO}$, and inflammatory cell infiltration [10]. Recent studies also have shown that rGO is likely to be toxic and could integrate cell membranes and induce programmed cell death in a dose-dependent manner, particularly in concentrations higher than $50 \mu \mathrm{g} / \mathrm{L}$ [11-13]. In order to address these issues and to improve the bioavailability of $\mathrm{rGO}$, it is essential to investigate its implications on the safety of living systems and develop a better understanding of toxicological mechanisms, which would facilitate the existing methods for rGO preparation (with minimal toxicity for safer biomedical applications).

The current study is motivated by the requirements for a better understanding of the mechanisms and in vitro efficacy of graphene-induced degradation of cells. The in vitro toxicity of rGO against two lung cancer cells, A549 and SKMES-1, has been assessed and compared for the first time without premodification of $\mathrm{rGO}$. We conducted the cell viability tests and measured the implications of early and late apoptosis and necrosis pathways to investigate the oxidative stress potential, and induction of apoptosis. We also showed the general trend between cell death rates and concentrations for cancer cells using a Gaussian process regression model. Our results demonstrated that a low concentration of rGO significantly produced late apoptosis and necrosis rather than early apoptotic events, though rGO was still able to disintegrate the cellular membranes in a dose dependent manner. Given the evolving field of graphene-based nanomedicine, our findings regarding the toxicity of graphene presented in this paper using in vitro models would play a significant role in paving a new way to future biomedical applications of graphene.

\section{Materials and methods:}

Synthesis and characterization: Exfoliated graphene oxide (GO) flakes were prepared following the modified Hummers method previously reported by us [14]. $2 \mathrm{~g}$ graphite flake, $1.5 \mathrm{~g} \mathrm{NaNO}_{3}$ and $150 \mathrm{ml} \mathrm{H}_{2} \mathrm{SO}_{4}$ (98\%) were added in an $800 \mathrm{ml}$ flask and mixed under magnetic stirring. The flask was immersed in an oil bath warmed to $35^{\circ} \mathrm{C}$, before $9 \mathrm{~g} \mathrm{KMnO}_{4}$ had been added into the flask. The mixture was continuously stirred for $24 \mathrm{~h}$, followed by further addition of $280 \mathrm{ml} \mathrm{H}_{2} \mathrm{SO}_{4}(5 \%)$ and increasing the temperature to $85-95 \stackrel{\circ}{\circ}$. After $2 \mathrm{~h}$ further stirring, the bath was removed and the flask 
was allowed to cool down to around $60{ }^{\circ} \mathrm{C}$ before further addition of $15 \mathrm{ml} \mathrm{H}_{2} \mathrm{O}_{2}(30$ wt\%) into it. After another $2 \mathrm{~h}$ stirring, the solid product in the suspension was collected, and washed repeatedly with diluted $\mathrm{HCl}$ (3 wt\%) and distilled water to remove any residual $\mathrm{Mn}^{4+}$ and other impurities.

The resultant GO was dispersed in water under stirring to the concentration of 0.25 $\mathrm{mg} \mathrm{mL}^{-1} .75 \mathrm{~mL}$ GO $(0.25 \mathrm{mg} / \mathrm{ml})$ was mixed with $1.5 \mathrm{~mL}$ hydrazine (35 wt\%) under magnetic stirring in a flask heated (in an oil bath) to $100^{\circ} \mathrm{C}$. The resultant $\mathrm{rGO}$ powder was then washed with distilled water for further characterization.

High resolution microstructural images were taken on a JEOL-2100 transmission electron microscope (TEM) operating at an accelerating voltage of $200 \mathrm{kV}$. TEM samples were prepared by ultrasonic dispersion of sample powder in acetone for 20 mins followed by dropping onto a holey carbon $\mathrm{Cu}$ grid using micropipette. X-ray diffraction (XRD) analysis was performed using Cu Ka radiation (at $40 \mathrm{kV}$ and $40 \mathrm{~mA}$ ). Spectra were collected at a scan rate of $(2 \theta) / \mathrm{min}$ and with a step size of $0.02^{\circ}(2 \theta)$. Fourier-transform infrared (FTIR) spectra were recorded in the wavenumber range of 4000-500 $\mathrm{cm}^{-1}$ using a Bruker Optics Tensor-27 FTIR spectrometer. Samples used in this case were prepared by mixing the original sample powders with $\mathrm{KBr}$. Raman spectra were recorded using a $532 \mathrm{~nm}$ laser excitation operating at $6 \mathrm{~mW}$ power. Zeta potential measurements were carried out using a colloidal dynamics zeta probe to identify the surface charge of $\mathrm{rGO}$ as a function of $\mathrm{pH}$, balanced in the acidic-basic ranges using $10^{-1} \mathrm{M} \mathrm{HCl}$ and $\mathrm{KOH}$ solutions, respectively.

Cell viability: A549 and SKMES-1 lung cancer cells were seeded in 6 well plates at a density of 350,000 per well. After overnight incubation, cells were treated with or without $5,50,250,500$ and $1000 \mu \mathrm{g} / \mathrm{ml}$ of rGO for $24 \mathrm{~h}$. Then, cells were trypsinised and centrifuged at $200 \mathrm{~g}$ for 5 minutes. Cell pellets were then re-suspended in $100 \mu \mathrm{l}$ of annexin binding buffer (BioLegend, UK) containing annexin (BioLegend, UK) and propidium iodide (PI) (Sigma-Aldrich, UK), and incubated for 15 minutes in the dark. Flow cytometry was performed using a Guava flow cytometer. The data was analysed using the Guava 3.1.1 software. The early and late apoptotic populations of the cells were analysed by flow cytometer Alexa Fluor647 Annexin V (apoptosis) - F2, Propidium iodide necrosis -F3 YEL. The criteria for early and late apoptotic cells are Annexin V-positive, PI-negative and Annexin V-positive, and PI-positive, respectively. 
Signals were detected using Alexa Fluor ${ }^{\circledR}$ 647, a bright far-red-fluorescent dye with excitation ideally suited for the $594 \mathrm{~nm}$ or $633 \mathrm{~nm}$ laser lines. PI is yellow-fluorescent dye with excitation ideally suited to the $532 \mathrm{~nm}$ laser line. Toxicity assays were repeated 3 times. The data were analysed in GraphPad Prism 5.04, and expressed as $\%$ cell count \pm SD, Mann Whitney. $P<0.05$ was considered statistically significant. The cell culture media were buffers that buffered the $\mathrm{pH}$ and maintained it at neutral level. The $\mathrm{pH}$ of the cell culture media were tested and collected from treated cells and no difference was found in the $\mathrm{pH}$ between basal media at 0 and $24 \mathrm{~h}$ after treatment.

Regression model: Additionally, we investigated the relationships between concentration of rGO and cell death rate, so as to determine the most appropriate concentration levels for biological applications. Standard non-linear regression methods may only capture the general trend without taking into account the uncertainties in measurements and predictions. We therefore used a Gaussian process (GP) to model the relationships and readily incorporate the uncertainties in measurements to produce a Bayesian posterior predictive distribution [15]. A GP is essentially a collection of random variables, and any finite number of these have joint Gaussian distribution. Given a dataset $D=\left\{\left(x_{i}, y_{i}\right)\right\}_{i=1}^{n}$ with $n$ data points (where $x_{i}$ is the $i$ th rGO concentration and $y_{i}$ is the associated cell count), the posterior predictive distribution is $P\left(y_{n+1} \mid x_{n+1}, D, \theta\right) \sim \aleph\left(y_{n+1} \mid \mu\left(x_{n+1}\right), \sigma\left(x_{n+1}\right)\right)$. Here, the mean prediction $\mu($.$) and the standard deviation \sigma($.$) are functions of the covariance matrix.$ The covariance matrix captures the covariance between observed concentrations, which is governed by the specific covariance function and the set of hyper-parameters $\theta$. In this study, we used the Matern52 covariance function. The hyper-parameters are set by maximising the likelihood of the data.

\section{Results and discussion:}

As well-known, reduction of $\mathrm{GO}$ in an aqueous suspension results in agglomerated graphene nanosheets [16]. Similarly to that reported previously [17,18], as-prepared rGO nanosheets from the present work also exhibited typical wrinkled and scrolled structures (Figure $1 \mathbf{a}, \mathbf{b}$ ). They consisted of a few-layers (typically 6-8 layers), and had an average thickness of $1.5 \mathrm{~nm}$, as revealed by HRTEM (Figure 1b). XRD 
(Figure2 A) further shows a diffraction peak at $26.40^{\circ}(2 \theta)$ corresponding to the (002) lattice plane of $\mathrm{rGO}$ with interlayer spacing of $3.37 \AA$, indicating the formation of $\mathrm{sp}^{2}$ network of carbon [19]. As shown in Figure 2B, rGO exhibited a D band at $1358 \mathrm{~cm}^{-1}$ and a $G$ band at $1595 \mathrm{~cm}^{-1}$, showing a series of defects and the in-plane stretching motion of pairs of $s p^{2}$ atoms, respectively [20]. FTIR peak at $3434 \mathrm{~cm}^{-1}$ was assigned to the $\mathrm{O}-\mathrm{H}$ stretching vibration (Figure $2 \mathrm{C}$ ). The weak peaks at $1622 \mathrm{~cm}^{-1}, 1399 \mathrm{~cm}^{-1}$, $1240 \mathrm{~cm}^{-1}$, and $1071 \mathrm{~cm}^{-1}$ arose from $\mathrm{C}=\mathrm{C}$ stretching vibration, $\mathrm{O}-\mathrm{H}$ deformation, $\mathrm{C}=\mathrm{O}$ (epoxy) stretching vibration, and $\mathrm{C}=\mathrm{O}$ (alkoxy) stretching, respectively, implying that the original functional groups were largely removed [21]. Zeta potential is a key parameter in the evaluation of stability of colloidal dispersions and prediction of the mobility/reaction of nanoparticles inside the cells [17]. Nanomaterials are generally considered to be fairly stable in a solution if the corresponding zeta potential is sufficiently high (more positive and negative than $+30 \mathrm{mV}$ and $-30 \mathrm{mV}$ respectively) [22]. As depicted in Figure 2D, as-prepared rGO nanosheets showed a maximal zeta potential of $-49.2 \mathrm{mV}$ at $\mathrm{pH} \mathrm{12,} \mathrm{which} \mathrm{was} \mathrm{resulted} \mathrm{from} \mathrm{the} \mathrm{reduction} \mathrm{of} \mathrm{different}$ functional groups existing on the surface of the original GO. 

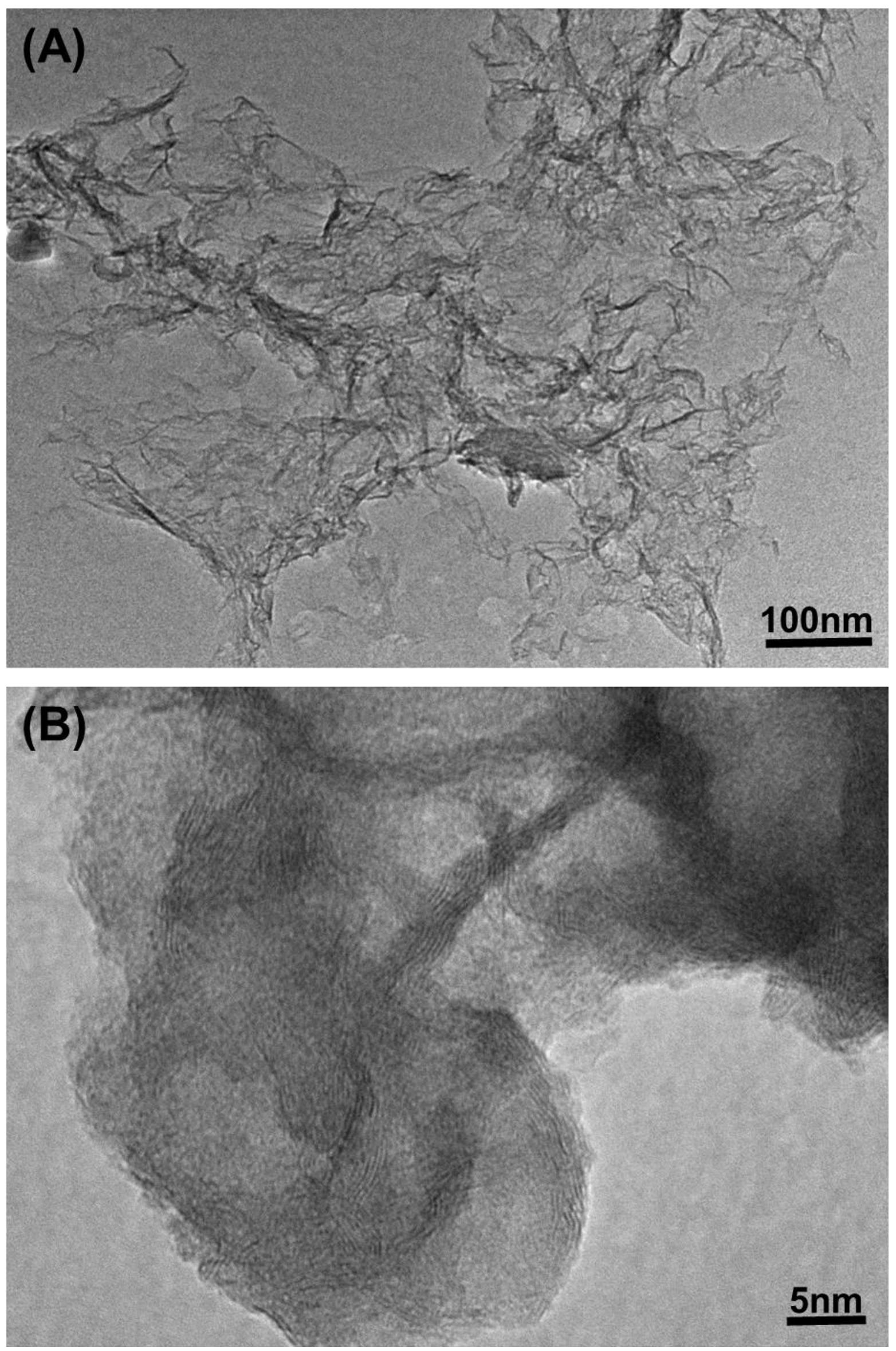

Figure 1: (A)-TEM and (B) HRTEM images of as-prepared exfoliated rGO sheets. 

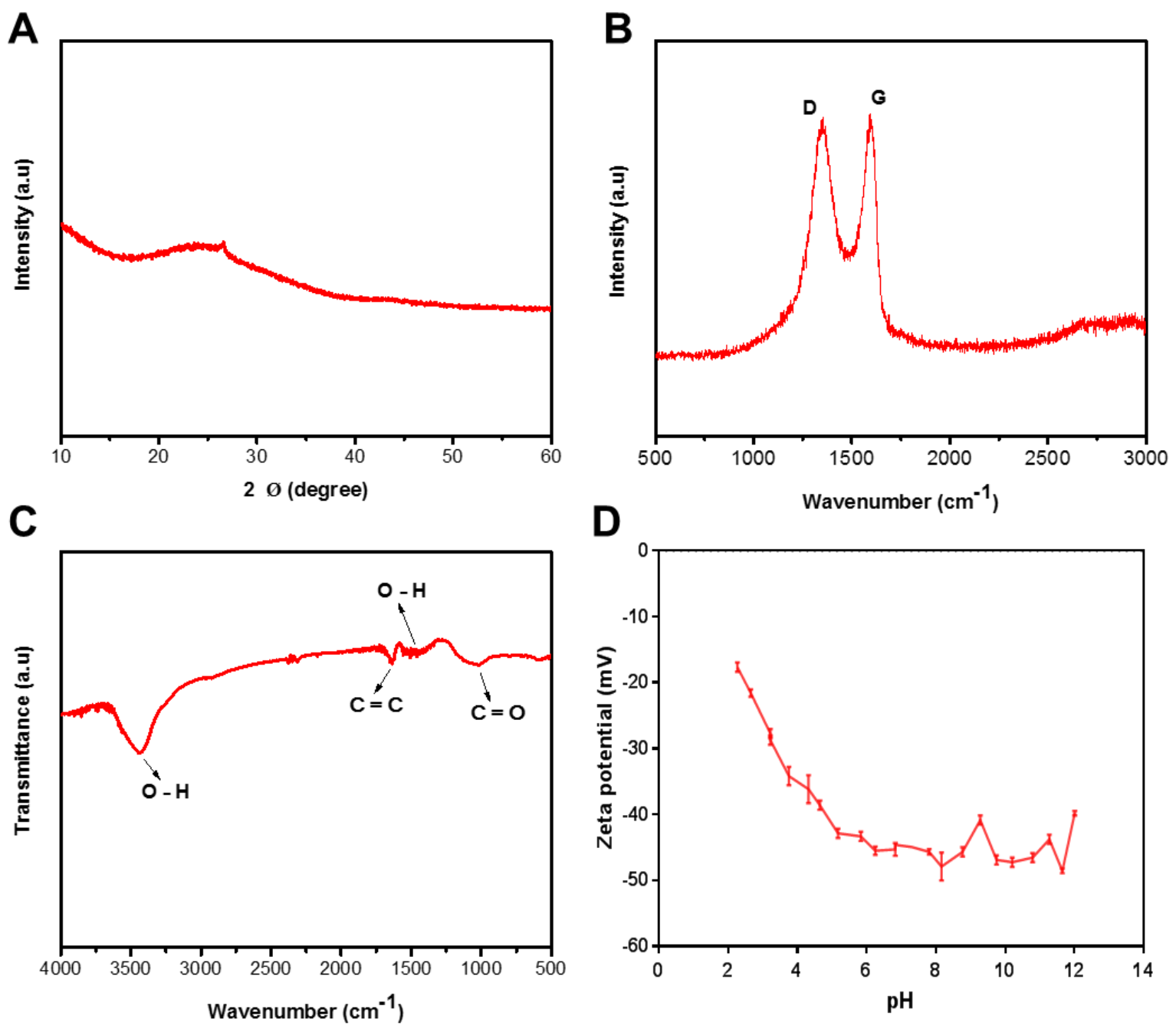

Figure 2: (A)- XRD pattern, (B)- Raman spectrum, (C) FTIR spectrum, and (D) Zeta potential-pH curve of as-prepared rGO.

As demonstrated in Figure 3A, after 24-h exposure to $\mathrm{GGO}$, the cell viability in the case of $A 549$ cells decreased by virtue of increasing the concentration of rGO from 5 to $1000 \mu \mathrm{g} / \mathrm{ml}$. For example, the percentage of living cells was reduced to 70,50 and $40 \%$ at concentrations 5,50 and $250 \mu \mathrm{g} / \mathrm{ml}$ respectively, compared to the controls ( 0 $\mu \mathrm{g} / \mathrm{ml}, \sim 90 \%)$. However, in SKMES-1 cells, rGO-induced toxicity was reduced significantly at a concentration of $50 \mu \mathrm{g} / \mathrm{ml}$ or above. Cell viability was reduced to 70 , 60,42 and $42 \%$ at concentrations of $50,250,500$ and $1000 \mu \mathrm{g} / \mathrm{ml}$, respectively, compared to the controls $(0 \mu \mathrm{g} / \mathrm{ml}, \sim 80 \%)$. Cells undergoing early apoptosis significantly increased when treated with $50 \mu \mathrm{g} / \mathrm{ml}$ in a dose dependent manner up to $500 \mu \mathrm{g} / \mathrm{ml}$ (both in A549 and SKMES-1 cells) (Figure 3B). A dose-dependent increase in late apoptosis (Figure 3C) and necrosis (Figure 3D) was also observed in both cell 
lines, where rGO demonstrated a greater toxic effect on A549 cells compared to SKMES- 1 cells.

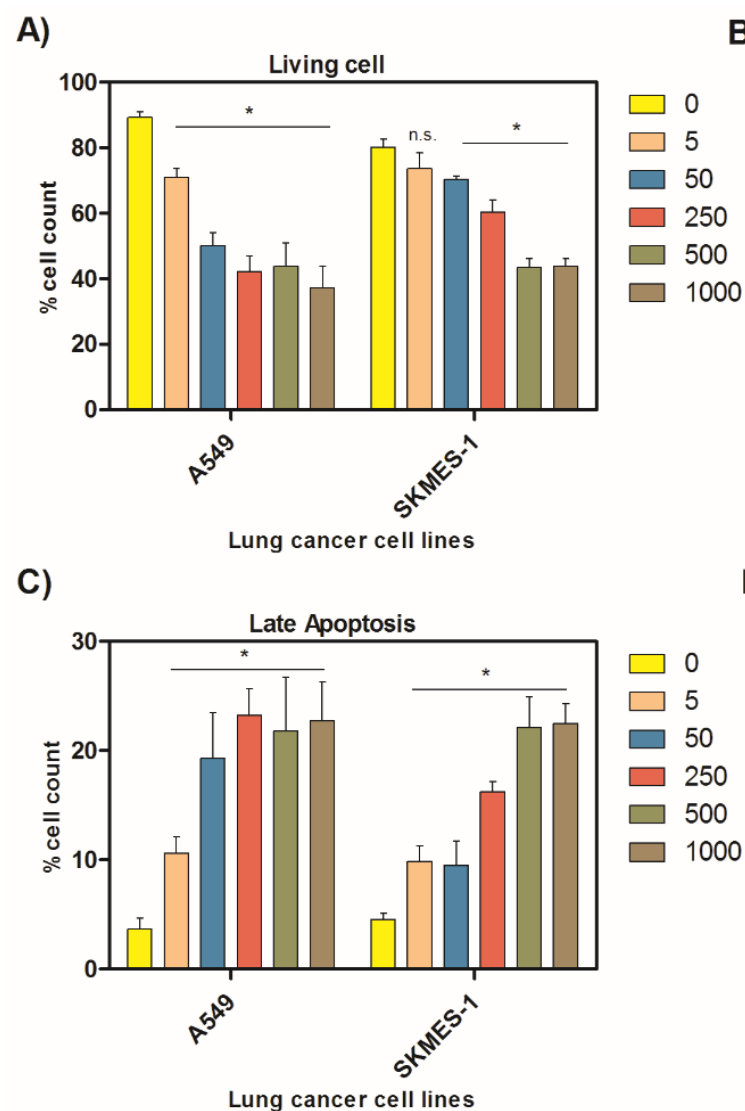

B)

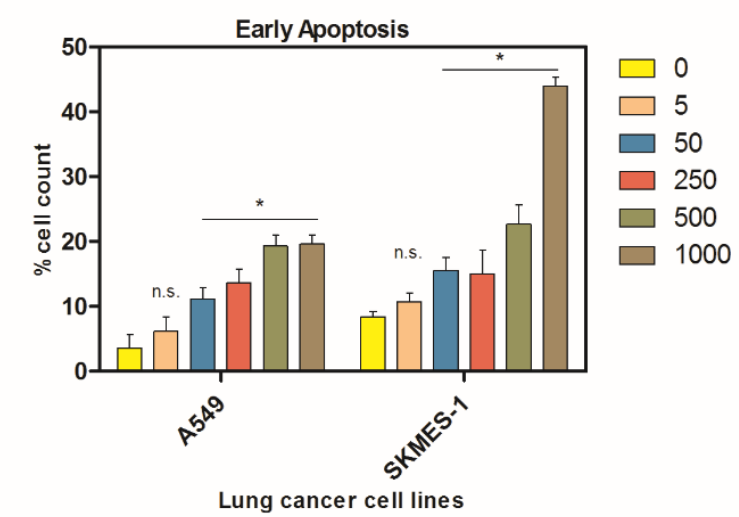

D)

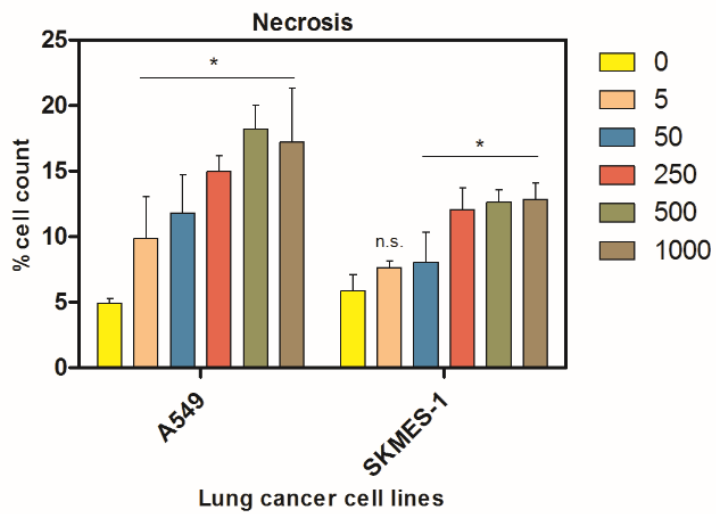

Figure 3. Bar graphs quantifying the percentage of dead, living, early-stage apoptotic, and late-stage apoptotic cells in response to different concentrations of reduced graphene oxide (rGO). Flow cytometry for A549 and SKMES-1 lung carcinoma cells stained with annexin $\mathrm{V}$ (apoptosis) and propidium iodide ( $\mathrm{PI}$; late apoptosis and necrosis) following $24 \mathrm{~h}$ of treatment with various concentrations of rGO $(0-1000$ $\mu \mathrm{g} / \mathrm{ml}$ ). (A) graphic representation of percentage of living cells (B) early apoptosis (C) necrosis, (D) late apoptosis (flow cytometry) in response to rGO. Data were represented as mean $\pm S D$, n.s., ${ }^{*} p<0.05$ vs control $(0 \mu \mathrm{g} / \mathrm{ml})$.

The resulting predictive distributions from the trained GP models for A549 and SKMES-1 cells are shown in Figure 4. The models not only capture the measurement noises, but also indicate how much confidence may be derived from the predictions through the associated standard deviation. Interestingly, the model for A549 cells indicates that concentrations below $200 \mu \mathrm{g} / \mathrm{ml}$ are likely to be better than higher 
concentrations. In contrast, the model for SKMES-1 cells, concentrations between 600 and 800 are likely to yield lower cell death. These predictions match with the experimental results presented in Figure 3.
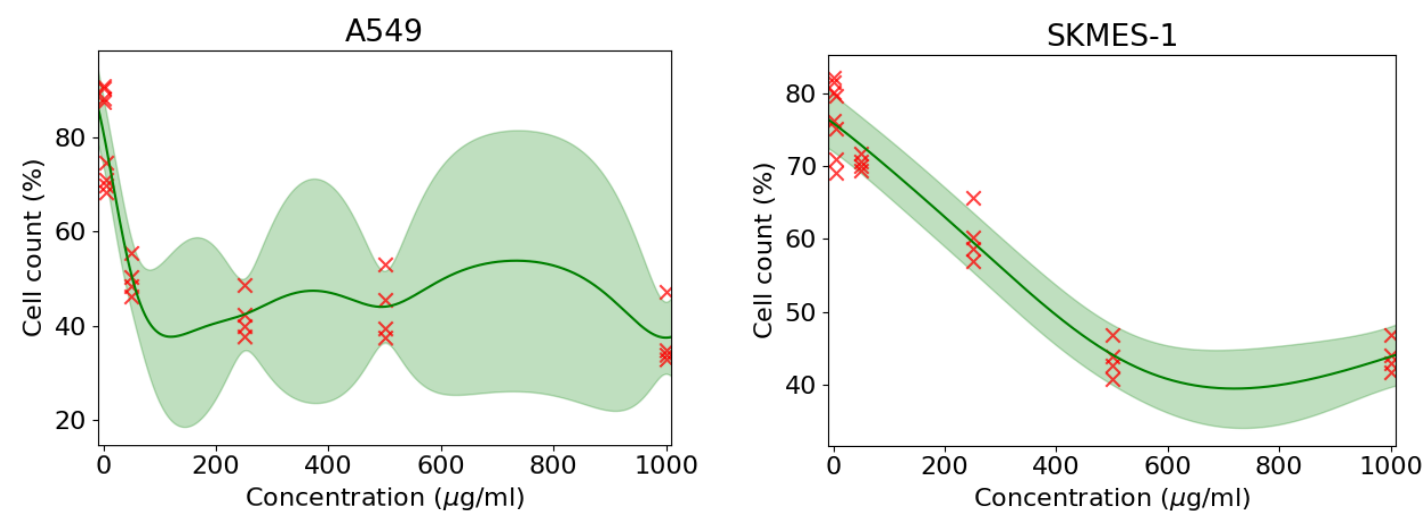

Figure 4. Gaussian process (GP) regression models for cell survival rates of A549 (left) and SKMES-1 (right) cells interacted with various concentrations of reduced Graphene Oxide ( $\mathrm{rGO}$ ). The green solid lines show the mean GP prediction, while the light green areas around the mean show the uncertainty (one standard deviation) in prediction. The models are trained with the data indicated by the red crosses.

Most interestingly, GP models can predict cell-specific toxicity levels of concentrations. As such, we may exploit this knowledge to run further experiments to find out optimal levels of concentrations. This approach is better known as Bayesian optimisation: a sequential design method that may locate near-optimal solutions with limited number of time consuming and computationally expensive experiments [23]. 

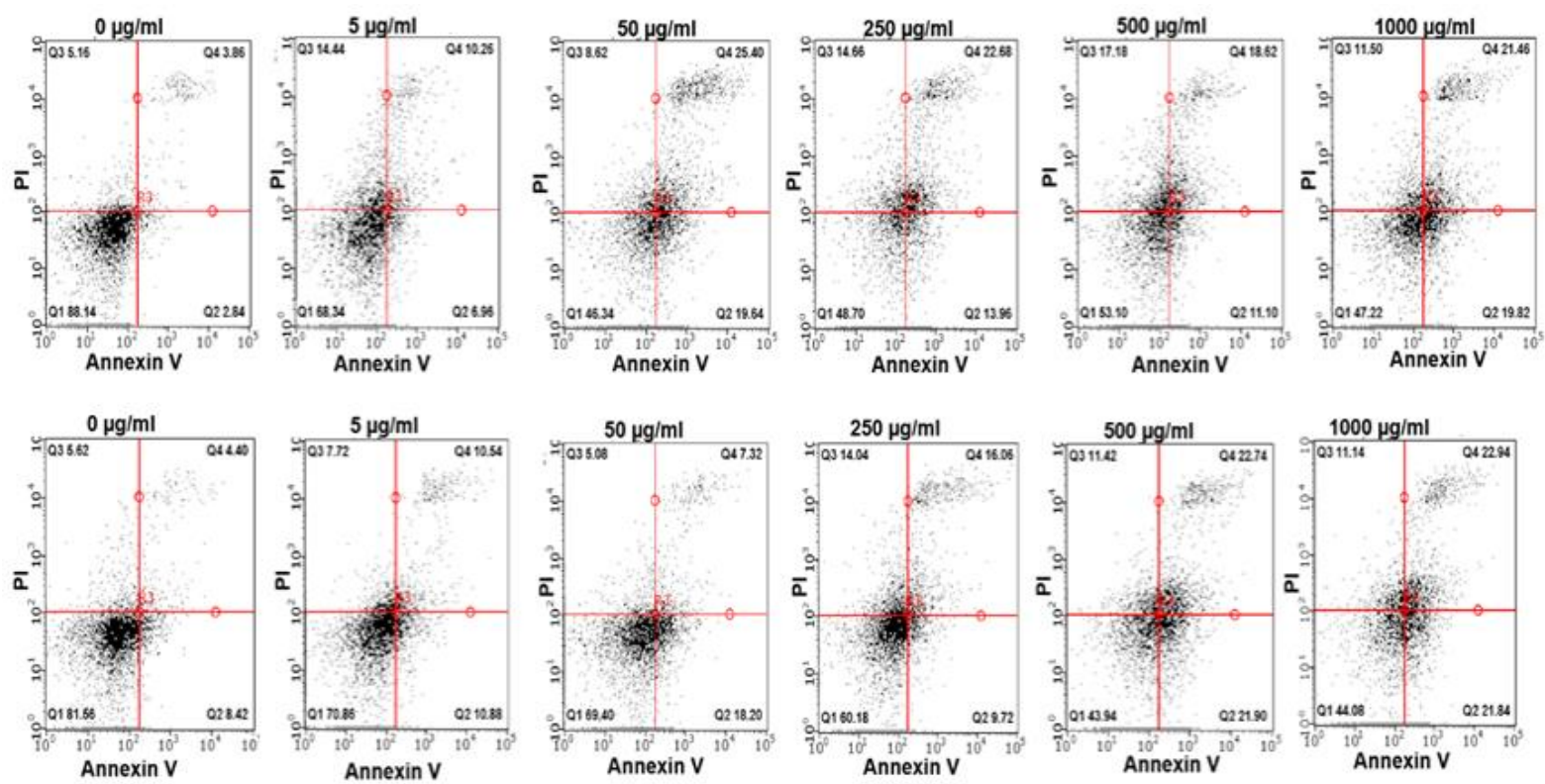

Figure 5: Representative FACS images and analysis of one experiment. Data were presented as percentage of the cell population. Cell viability of A549 (upper panel) and SKMES-1 (lower panel) at selected concentrations. Experiments were performed and interpreted as follows: Annexin $\mathrm{V}^{\text {-ve }} / \mathrm{PI}^{\text {-ve }}$ cells (lower left quadrant), $\mathrm{AnnV}^{+\mathrm{ve}} / \mathrm{PI}^{\text {-ve }}$ cells (lower right quadrant), $\mathrm{AnnV}^{+\mathrm{ve}} / \mathrm{PI}^{+v e}$ (upper right quadrant) and $\mathrm{AnnV}^{-v e} / \mathrm{PI}^{+v e}$ (upper left quadrant) were considered as living, early apoptotic, late apoptotic, and necrotic cells respectively.

Cell viability, early and late apoptosis and necrosis were also measured under similar conditions to those mentioned above. Early apoptosis is typically defined by an increase in phosphatidylserine (PS) expression on an intact cell membrane (detected by annexin V). In late apoptosis, however the membrane loses its integrity allowing PI into the cell and flags these cells as late apoptotic/necrotic. This study showed that rGO induced apoptosis and necrosis above the concentration of $5 \mu \mathrm{g} / \mathrm{ml}$ in A549 and above the concentration of $50 \mu \mathrm{g} / \mathrm{ml}$ in SKMES-1 cell lines (Figure 5). Even at lower concentrations, rGO was shown to significantly produce late apoptosis and necrosis, suggesting that $\mathrm{rGO}$ was able to disintegrate cellular membranes (PI staining) at lower concentrations. Interestingly, the effect was more pronounced in A549 cells compared to SKMES-1 cells upon exposure of rGO. Kumar et al [11] recently reported the high toxicity of rGO on A549 cancer cells arising from its reduced lateral size, and showed alteration of mitochondrial homeostasis upon rGO exposure. Hu et al. [10] found that 
rGO caused a dose-dependent decrease in A549 cell viability to $47 \%(20 \mathrm{mg} / \mathrm{ml})$ and $15 \%(85 \mathrm{mg} / \mathrm{ml})$. Toxicity screening of engineered nanomaterials is always accomplished in concentration-dependent manner to develop safety profile and risk management strategies for their real-world applications. In the case of graphene, low concentrations are generally not toxic in mammalian cells but high concentrations play a role in plasma membrane internalization and induction of programmed cell death [24]. Liao et al. [25] reported toxicity of graphene sheets in dose-dependent manner which showed chronic hemolysis activity to suspended erythrocytes owing to its good electrostatic interactions with the erytrocyte membrane. Also high concentrations of graphene sheets $(200 \mu \mathrm{g} / \mathrm{ml})$ produced higher reactive oxygen species in human skin fibroblast cells than low concentrations $(3.125 \mu \mathrm{g} / \mathrm{ml})$ of graphene sheets due to their strong interaction and binding to the cell surface. Comparable results were reported in A549 cell line $[10,11]$ suggesting that higher concentrations of graphene sheets damage membrane integrity and block the localization of sheets with cell barriers and produce high yield of reactive oxygen species. rGO has pronounced effects on cellular viability, oxidative stress, and cell death compared to GO because of its sharp edges, functional groups, surface charge and nanosheets which facilitate its improved cellular uptake [26]. The combined effect of early and late apoptosis and necrosis events produced by rGO implies a threat to clinical utility of rGO. rGO induced toxicity potentially causes the poor delivery of essential nutrients to cancer cells by blocking the immune tolerance of the host cells to recruit blood vessel factories for their survival. Oxidative stress is one of the key paradigms leading to graphene toxicology that reduces the viability of cells and also hinders the uptake of essential proteins and nutrients into cells $[12,27]$. Production and abolition of reactive oxygen species are well-adjusted inside the cells, and altering the balance could induce lipid peroxidation, dysfunction of mitochondria, and apoptosis and necrosis [28]. The toxic transformation of graphene, irrespective of the specific structure/assembly of graphene used, relies on its bioaccumulation, the structural and chemical morphology of graphene as well as the generation of reactive oxygen species in both dark and photo toxicity environments [29]. The excessive reactive oxygen species generation may induce the mitochondrial membrane damage from lipid peroxidation, DNA damage and apoptosis (at low dose) [30]. The generation of reactive oxygen species to induce oxidative stresses is considered to be a leading cause of toxicity for graphene nanocomposites [28]. Furthermore, the rGO revealed necrosis was more profound and prevalent at 
high doses, which is likely due to gene deregulation and encoding, demolition phase of apoptosis process, whereas, apoptosis events induced by low dose of rGO might be triggered due to death-receptor medicated pathways and mitochondrial-driven intrinsic pathways [31]. Furthermore, based on the existing literature work and the present study, it is revealed that cellular membrane distress, oxidative stresses and direct contact of the sharp edges with the cells are considered to be majorly responsible for the toxicity of rGO. Direct contact of sharp edges and lateral dimensions of rGO may induce genotoxic lesions and genomic instability through their interactions with the DNA sequence and structure in target cells [32]. In addition, the presence of impurities and toxic chemicals during the fabrication of graphene nanocomposites may have adverse effects on their bioavailability to living systems. Diversity in size, shape, surface chemistry, lateral dimensions and fabrication routes of rGO make it impossible to establish clearly the comparison of biological and toxicological impacts of rGO between different studies. As different preparation methods produce different quantities of functional groups and free radicals on the surface of rGO, this subsequently induces oxidative stresses. Therefore, terminology, nomenclature and preparation methods need to be reconciled and standardized to validate analytical methods for measuring toxicology impacts, bio distribution and physicochemical characteristics of rGO in living systems. With the rapid growth and expansion of the graphene market, it is necessary to assess the risk management strategies related to the fabrication processes and clinical settings which can potentially minimise the environmental and clinical risks of graphene. Furthermore, an important benefit of graphene over other nano-assemblies is that its physiochemical properties such as hydrophobicity/hydrophilicity, surface charge, size, and surface area could be tuned by adjusting synthesis conditions. Moreover, a variety of postpreparation methods may be introduced to graphene sheets targeting the efficient reduction of graphene oxide. Surface functionalization, reduction strategies, doping, and introduction of biocompatible coatings are another promising and intriguing window of opportunity to improve the bioavailability of rGO to living systems. This is of a particular importance in relation to bio-persistence and long-term toxicity of this material, since there is a lack of long term in vivo monitoring in this area.

The results from this study confirmed that $\mathrm{rGO}$ poses higher biological risks than GO and other derivatives of graphene. In order to improve the bioavailability of 
rGO, several significant challenges remain to be addressed such as translating its toxicological mechanisms and preparation of safer and modified rGO sheets. Further toxicological studies should take into consideration the facile preparation of the sample such as the intermixing of debris from sample impurities, residues of strong acids and reducing agents, which may profoundly revise and improve the surface features of rGO. Further in vivo investigations are also required to trace the bioavailability of rGO and to clarify the clinical effects of this 'miracle material'.

\section{Conclusion}

This paper reports the in vitro toxic effects of rGO on lung cancer cells (A549 and SKMES-1) as a function of its concentration. The results indicated that rGO caused significant late apoptosis and necrosis rather than early apoptotic event at lower concentrations, suggesting that $\mathrm{rGO}$ was able to disintegrate the cellular membranes in a dose dependent toxicity manner. For the toxicity exposures undertaken, late apoptosis and necrosis occurred, which was likely resultant from the limited bioavailability of unmodified rGO in lung cancer cells.

\section{Acknowledgement}

This work was supported by EPSRC Centre for Doctoral Training in Metamaterials, XM² [Grant no. EP/L015331/1] the University Of Exeter EX4, United Kingdom, FORCE Cancer Charity [Grant No. 50703] United Kingdom, Higher Committee for Education Development (HCED), Iraq, and the EPSRC project on Data Driven Surrogate-Assisted Evolutionary Fluid Dynamic Optimisation [grant no. EP/M017869/1], University of Exeter, United Kingdom.

\section{References}

[1] Graphene market size global forecast http://www.marketsandmarkets.com/PressReleases/graphene-electronics.asp and http://www.marketsandmarkets.com/PressReleases/graphene.asp

[2] Santos, C. M., Mangadlao, J., Ahmed, F., Leon, A., Advincula, R. C., \& Rodrigues, D. F. (2012). Graphene nanocomposite for biomedical applications: fabrication, antimicrobial and cytotoxic investigations. Nanotechnology, 23(39), 395101. 
[3] Nguyen, B. H., \& Nguyen, V. H. (2016). Promising applications of graphene and graphene-based nanostructures. Advances in Natural Sciences: Nanoscience and Nanotechnology, 7(2), 023002.

[4] Ou, L., Song, B., Liang, H., Liu, J., Feng, X., Deng, B., \& Shao, L. (2016). Toxicity of graphene-family nanoparticles: a general review of the origins and mechanisms. Particle and fibre toxicology, 13(1), 57.

[5] Liu, S., Zeng, T. H., Hofmann, M., Burcombe, E., Wei, J., Jiang, R., \& Chen, Y. (2011). Antibacterial activity of graphite, graphite oxide, graphene oxide, and reduced graphene oxide: membrane and oxidative stress. ACS nano, 5(9), 6971-6980.

[6] Sasidharan, A., Panchakarla, L. S., Chandran, P., Menon, D., Nair, S., Rao, C. N. R., \& Koyakutty, M. (2011). Differential nano-bio interactions and toxicity effects of pristine versus functionalized graphene. Nanoscale, 3(6), 2461-2464.

[7] Syama, S., Aby, C. P., Maekawa, T., Sakthikumar, D., \& Mohanan, P. V. (2017). Nano-bio compatibility of PEGylated reduced graphene oxide on mesenchymal stem cells. 2D Materials, 4(2), 025066.

[8] Tabish, T. A., Chabi, S., Ali, M., Xia, Y., Jabeen, F., \& Zhang, S. (2017). Tracing the Bioavailability of Three-Dimensional Graphene Foam in Biological Tissues. Materials, 10(4), 336.

[9] Zhang, X., Nan, X., Shi, W., Sun, Y., Su, H., He, Y., \& Ge, D. (2017). Polydopaminefunctionalized nanographene oxide: a versatile nanocarrier for chemotherapy and photothermal therapy. Nanotechnology, 28(295102), 295102.

[10] Hu, W., Peng, C., Luo, W., Lv, M., Li, X., Li, D., \& Fan, C. (2010). Graphene-based antibacterial paper. ACS nano, 4(7), 4317-4323.

[11] Mittal, S., Kumar, V., Dhiman, N., Chauhan, L. K. S., Pasricha, R., \& Pandey, A. K. (2016). Physico-chemical properties based differential toxicity of graphene oxide/reduced graphene oxide in human lung cells mediated through oxidative stress. Scientific reports, 6, 39548.

[12] Volkov, Y., McIntyre, J., \& Prina-Mello, A. (2017). Graphene toxicity as a doubleedged sword of risks and exploitable opportunities: a critical analysis of the most recent trends and developments. 2D Materials, 4(2), 022001.

[13] Seabra, A. B., Paula, A. J., de Lima, R., Alves, O. L., \& Durán, N. (2014). Nanotoxicity of graphene and graphene oxide. Chemical research in toxicology, 27(2), 159-168.

[14] Lin, L., Zheng, X., Zhang, S., \& Allwood, D. A. (2014). Surface energy engineering in the solvothermal deoxidation of graphene oxide. Advanced Materials Interfaces, 1(3).

[15] Rasmussen, C. E., \& Williams, C. K. (2006). Gaussian processes for machine learning (Vol. 1). Cambridge: MIT press.

[16] Li, D., Müller, M. B., Gilje, S., Kaner, R. B., \& Wallace, G. G. (2008). Processable aqueous dispersions of graphene nanosheets. Nature nanotechnology, 3(2), 101-105. [17] Lee, S., Bong, S., Ha, J., Kwak, M., Park, S. K., \& Piao, Y. (2015). Electrochemical deposition of bismuth on activated graphene-nafion composite for anodic stripping voltammetric determination of trace heavy metals. Sensors and Actuators B: Chemical, 215, 62-69.

[18] Pham, V. H., Pham, H. D., Dang, T. T., Hur, S. H., Kim, E. J., Kong, B. S., ... \& Chung, J. S. (2012). Chemical reduction of an aqueous suspension of graphene oxide by nascent hydrogen. Journal of Materials Chemistry, 22(21), 10530-10536.

[19] Sykam, N., \& Rao, G. M. (2017). Room temperature synthesis of reduced graphene oxide nanosheets as anode material for supercapacitors. Materials Letters. 
[20] Díez-Betriu, X., Álvarez-García, S., Botas, C., Álvarez, P., Sánchez-Marcos, J., Prieto, C., \& de Andrés, A. (2013). Raman spectroscopy for the study of reduction mechanisms and optimization of conductivity in graphene oxide thin films. Journal of Materials Chemistry C, 1(41), 6905-6912.

[21] Ren, P. G., Yan, D. X., Ji, X., Chen, T., \& Li, Z. M. (2010). Temperature dependence of graphene oxide reduced by hydrazine hydrate. Nanotechnology, 22(5), 055705.

[22] Konkena, B., \& Vasudevan, S. (2012). Understanding aqueous dispersibility of graphene oxide and reduced graphene oxide through $\mathrm{p} \mathrm{K}$ a measurements. The journal of physical chemistry letters, 3(7), 867-872.

[23] Shahriari, B., Swersky, K., Wang, Z., Adams, R. P., \& de Freitas, N. (2016). Taking the human out of the loop: A review of bayesian optimization. Proceedings of the IEEE, 104(1), 148-175.

[24] Zhang, B., Wei, P., Zhou, Z., \& Wei, T. (2016). Interactions of graphene with mammalian cells: Molecular mechanisms and biomedical insights. Advanced drug delivery reviews, 105, 145-162.

[25] Liao, K. H., Lin, Y. S., Macosko, C. W., \& Haynes, C. L. (2011). Cytotoxicity of graphene oxide and graphene in human erythrocytes and skin fibroblasts. ACS applied materials \& interfaces, 3(7), 2607-2615.

[26] O. Akhavan, \& E. Ghaderi, ACS nano, 4(10), (2010), pp. 5731-5736.

[27] Montagner, A., Bosi, S., Tenori, E., Bidussi, M., Alshatwi, A. A., Tretiach, M., \& Syrgiannis, Z. (2016). Ecotoxicological effects of graphene-based materials. 2D Materials, 4(1), 012001.

[28] Palmieri, V., Lauriola, M. C., Ciasca, G., Conti, C., De Spirito, M., \& Papi, M. (2017). The graphene oxide contradictory effects against human pathogens. Nanotechnology, 28(15), 152001.

[29] Montagner, A., Bosi, S., Tenori, E., Bidussi, M., Alshatwi, A. A., Tretiach, M., \& Syrgiannis, Z. (2016). Ecotoxicological effects of graphene-based materials. 2D Materials, 4(1), 012001.

[30] Lopez, E., Arce, C., Oset-Gasque, M. J., Canadas, S., \& Gonzalez, M. P. (2006). Cadmium induces reactive oxygen species generation and lipid peroxidation in cortical neurons in culture. Free Radical Biology and Medicine, 40(6), 940-951.

[31] Wojtoniszak, M., Chen, X., Kalenczuk, R. J., Wajda, A., Łapczuk, J., Kurzewski, M., \& Borowiak-Palen, E. (2012). Synthesis, dispersion, and cytocompatibility of graphene oxide and reduced graphene oxide. Colloids and Surfaces B: Biointerfaces, 89, 79-85.

[32] Akhavan, O., Ghaderi, E., \& Akhavan, A. (2012). Size-dependent genotoxicity of graphene nanoplatelets in human stem cells. Biomaterials, 33(32), 8017-8025. 